Structure and spin density of ferric low-spin heme complexes determined with high-resolution ESEEM experiments at $35 \mathrm{GHz}$

Journal Article

Author(s):

García-Rubio, Inés; Mitrikas, George

Publication date:

2010-08

Permanent link:

https://doi.org/10.3929/ethz-b-000156488

Rights / license:

In Copyright - Non-Commercial Use Permitted

Originally published in:

JBIC Journal of Biological Inorganic Chemistry 15(6), https://doi.org/10.1007/s00775-010-0655-9 


\title{
Structure and spin density of ferric low-spin heme complexes determined with high-resolution ESEEM experiments at $35 \mathrm{GHz}$
}

\author{
Inés García-Rubio • George Mitrikas
}

Received: 9 December 2009/Accepted: 21 March 2010/Published online: 21 April 2010

(C) SBIC 2010

\begin{abstract}
The wide use of the heme group by nature is a consequence of its unusual "electronic flexibility." Major changes in the electronic structure of this molecule can result from small perturbations in its environment. To understand the way the electronic distribution is dictated by the structure of the heme site, it is extremely important to have methods to reliably determine both of them. In this work we propose a way to obtain this information in ferric low-spin heme centers via the determination of $\boldsymbol{g}, \boldsymbol{A}$, and $\boldsymbol{Q}$ tensors of the coordinated nitrogens using electron spin echo envelope modulation experiments at Q-band microwave frequencies. The results for two bisimidazole heme model complexes, namely, PPIX $(\operatorname{Im})_{2}$ and $\mathrm{CPIII}(\operatorname{Im})_{2}$, where PPIX is protoporphyrin IX, CPIII is coproporphyrin III, and Im is imidazole, selectively labeled with ${ }^{15} \mathrm{~N}$ on the heme or imidazole nitrogens are presented. The planes of the axial ligands were found to be parallel and oriented approximately along one of the $\mathrm{N}-\mathrm{Fe}-\mathrm{N}$ directions of the
\end{abstract}

Electronic supplementary material The online version of this article (doi:10.1007/s00775-010-0655-9) contains supplementary material, which is available to authorized users.

I. García-Rubio ( $\square)$

Laboratory of Physical Chemistry, ETH Zürich,

Wolfgang Pauli-Strasse 10,

8093 Zurich, Switzerland

e-mail: garciarubio@phys.chem.ethz.ch

G. Mitrikas

Institute of Materials Science,

NCSR "Demokritos",

15310 Athens, Greece

e-mail: mitrikas@ims.demokritos.gr slightly ruffled porphyrin ring (approximately $10^{\circ}$ ). The spin density was determined to reside in an iron $d$ orbital perpendicular to the heme plane and oriented along the other porphyrin $\mathrm{N}-\mathrm{Fe}-\mathrm{N}$ direction, perpendicular to the axial imidazoles. The benefit of the method presented here lies in the use of Q-band microwave frequencies, which improves the orientation selection, results in no/fewer combination lines in the spectra, and allows separation of the contributions of hyperfine and quadrupole interactions due to the fulfillment of the exact cancellation condition at $g_{Z}$ and the possibility of performing hyperfine decoupling experiments at the $g_{X}$ observer position. These experimental advantages make the interpretation of the spectra straightforward, which results in precise and reliable determination of the structure and spin distribution.

Keywords Low-spin heme .

Hyperfine sublevel correlation spectroscopy ·

Heme protein $\cdot$ Electron structure $\cdot$ Ferric iron

$\begin{array}{ll}\text { Abbreviations } \\ \text { CPIII } & \begin{array}{l}\text { Coproporphyrin III } \\ \text { CW }\end{array} \\ \text { DEFENCE } & \begin{array}{l}\text { Continuous wave } \\ \text { Deadtime-free electron spin echo envelope } \\ \text { modulation nuclear coherence-transfer } \\ \text { echoes }\end{array} \\ \text { dq } & \begin{array}{l}\text { Double quantum } \\ \text { ENDOR }\end{array} \\ \text { EPR } & \text { Electron-nuclear double resonance } \\ \text { ESEEM } & \text { Electron spin echo envelope modulation } \\ \text { HYSCORE } & \text { Hyperfine sublevel correlation spectroscopy } \\ \text { LS } & \text { Low spin } \\ \text { PPIX } & \text { Protoporphyrin IX } \\ \text { sq } & \text { Single quantum }\end{array}$




\section{Introduction}

Low-spin (LS) iron porphyrins are one of the most common prosthetic groups found in nature and constitute the active site of numerous heme proteins implicated in a whole variety of biochemical processes, a lot of them involving changes in the redox state [1,2]. For this reason iron porphyrin systems have been and still are the topic of numerous investigations. Many relevant attributes of their biochemical functions are determined by their structure and electron distribution; therefore, the investigation of these properties is essential to the understanding of the reaction mechanism. Especially interesting is to examine the unpaired electron density involved in a reaction of electron transfer, and even more to attempt to recognize the structural factors that govern the unpaired electron distribution by combining structural and electronic information. In this respect, electron paramagnetic resonance (EPR) spectroscopy and advanced methods such as electronnuclear double resonance (ENDOR) and electron spin echo envelope modulation (ESEEM) have yielded unique information on ferric LS heme systems [3] as they selectively probe the unpaired electron and its surroundings.

The distribution of the unpaired electron within the $3 d$ orbitals of $\mathrm{Fe}$ (III) can be obtained from the $g$ values measured in a continuous-wave (CW) EPR spectrum [4-6], but determining the orientation of these orbitals in disordered samples requires more effort. Dipolar interactions between electron and proton nuclear spins depend on the position of the proton relative to the iron; therefore, one can use the proton hyperfine couplings obtained by ENDOR [7-12] or ESEEM [8, 13-17] to determine the relative orientations between the $g$ matrix, heme molecular frame, and axial ligands. The main difficulty with this approach is that typically there are many protons in the active site and proton signals often cannot be unambiguously assigned.

The use of the nuclear quadrupole interaction of nitrogens directly coordinating the iron can also provide this information and lacks many of the complications mentioned above since there are only up to six nitrogens, the principal axis system of the nuclear quadrupole tensor coincides with the molecular axes, and the approximate octahedral symmetry of the coordination simplifies the assignment. X-band pulse-EPR experiments and in particular ESEEM spectroscopy have been used to find the relative orientation between the principal axis system of the nuclear quadrupole tensor $(\boldsymbol{Q})$ of the heme and axial ligand ${ }^{14} \mathrm{~N}$ and the ones of the $g$ tensor $[13,18,19]$, which are in turn related to the orientation of the orbital containing the unpaired electron via the counterrotation principle [20-22]. The problem with this approach is the difficulty in analyzing the spectra taken at the $g_{X}$ observer position which are most informative with respect to tensor orientations.
The assignment of the signals is impeded by the presence of combination lines and assuming the nuclear frequencies are successfully extracted, they cannot be interpreted in a straightforward way as they are very sensitive to a number of unknown parameters dependent on both nuclear quadrupole and hyperfine interactions.

In this work we show how the combination of hyperfine sublevel correlation spectroscopy (HYSCORE) and hyperfine decoupling experiments performed at Q-band $(35 \mathrm{GHz})$ can overcome these obstacles to obtain the distribution of unpaired spin and the orientation of nitrogencontaining axial ligands in a precise and reliable way. The higher nuclear Zeeman interaction achieved at Q-band microwave frequencies has two consequences. First, the exact cancellation condition at the observer position $g=g_{Z}$ is fulfilled in a good approximation, which allows the principal values of the $\boldsymbol{Q}$ tensors to be obtained by performing experiments at this field position. On the other hand, for the observer position $g=g_{X}$ the dominant interaction is the nuclear Zeeman interaction, which sets the nitrogen hyperfine interactions well in the weak coupling regime, where the spectra contain fewer combination lines and can be accurately analyzed also by performing numerical calculations. In addition to increasing the microwave frequency, the interpretation of nuclear frequency spectra can be simplified by eliminating the hyperfine interaction altogether through a decoupling procedure that employs strong and prolonged microwave pulses. During microwave irradiation, the local field at the nucleus generated by the electron spin is averaged to zero and so is the hyperfine interaction [23, 24].

The performance of decoupling experiments together with the strong nuclear Zeeman interaction and the better orientation selection achieved at Q-band at this field position result in a much simpler, more straightforward, and more reliable interpretation of the spectra that we expect this method to yield also good results in heme proteins and other complexes prepared in lower concentrations and lacking isotopic labeling. Since the principal values of $\boldsymbol{Q}$ are not expected to change significantly among similar LS heme systems, the values obtained in this work could be used to find the orientation information with the minimal number of experiments performed at the $g_{X}$ observer position.

\section{Materials and methods}

\section{Sample preparation}

LS heme model complexes were prepared by dissolving either iron(III) protoporphyrin IX (PPIX) chloride (from Sigma, purity more than $80 \%$ ) or iron(III) ${ }^{15} \mathrm{~N}$-coproporphyrin III $\left(\left[{ }^{15} \mathrm{~N}\right] \mathrm{CPIII}\right)$ chloride $\left({ }^{15} \mathrm{~N}\right.$ content higher than 
$99 \%$, Porphyrin Products) in a 1:1 mixture of dimethyl sulfoxide and chloroform. Subsequently, a tenfold excess of imidazole was added (either natural abundance imidazole or ${ }^{15} \mathrm{~N}$-imidazole, both from Aldrich Chemical, purity more than 99\%). The final concentration of the iron complex was about $10 \mathrm{mM}$ and the coordination of two molecules of imidazole to the porphyrin ring was evidenced by a change in color of the solution to a bright red. After preparation, the solutions were transferred to quartz tubes, frozen, and stored in liquid nitrogen until use.

\section{Spectroscopy}

Q-band EPR measurements were carried out with a homebuilt pulse spectrometer working in the $34.5-35.5-\mathrm{GHz}$ microwave frequency range [25] and equipped with an Oxford Instruments CF 935 helium gas-flow cryostat. All measurements were performed at a temperature of $6 \mathrm{~K}$ with a home-built resonator [26] that allows for sample diameters of $3.8 \mathrm{~mm}$.

HYSCORE experiments [27, 28] were performed using the pulse sequence $\pi / 2-\tau-\pi / 2-t_{1}-\pi-t_{2}-\pi / 2-\tau$-echo with pulse lengths $t_{\pi / 2}=24 \mathrm{~ns}$ and $t_{\pi}=16 \mathrm{~ns}$. The time intervals $t_{1}$ and $t_{2}$ were varied in steps of $16,24,32$, or $48 \mathrm{~ns}$. Spectra with several $\tau$ values were measured to avoid blind-spot effects (typically $\tau$ was 120,168 , and $232 \mathrm{~ns}$ ) and an eight-step phase cycle was used to eliminate unwanted echoes [28]. The time traces were baseline-corrected using a second-order polynomial, windowed with a Hamming function and zero-filled to 1,024 points. Then, a 2D Fourier transform was applied and the absolute-value spectrum was calculated. The result is a $2 \mathrm{D}$ pattern that correlates nuclear frequencies of the two different spin manifolds.

HYSCORE experiments were carried out at different values of the magnetic field that correspond to different orientations of the molecules with respect to the magnetic field (orientation selectivity). In particular, observer positions corresponding to the principal values of the $g$ matrix $\left(g_{X}, g_{Y}, g_{Z}\right)$ were systematically studied.

Hyperfine decoupling experiments [23, 24] were performed with a modified version of the 2D deadtime-free ESEEM nuclear coherence-transfer echoes (DEFENCE) sequence, $\pi / 2-\tau-p_{\mathrm{dec}}-t_{1}-\pi-t-p_{\mathrm{dec}}-\tau-$ echo, where the interpulse delay $t$ is varied. The second and fourth pulses (decoupling pulses) are strong and have equal but variable length, $T_{\mathrm{dec}}$; this introduces a second dimension along which the hyperfine-decoupled frequencies can be obtained. The pulse lengths were $t_{\pi / 2}=24 \mathrm{~ns}$ and $t_{\pi}=16 \mathrm{~ns} ; T_{\mathrm{dec}}$ was varied from $16 \mathrm{~ns}$ to $4.4 \mu \mathrm{s}$ in steps of $8 \mathrm{~ns}$. The time interval $t$ was swept in steps of 24 or $16 \mathrm{~ns}$. The $t_{1}$ value was set to $112 \mathrm{~ns}$ and was optimized by performing a series of 1D DEFENCE experiments [29]. For every experiment the powers of the $\pi$ and $\pi / 2$ pulses were appropriately set by optimizing the intensity of a two-pulse echo and the decoupling pulses were generated using a microwave field strength of $\omega_{1} / 2 \pi=32 \mathrm{MHz}$. Also, an eight-step phase cycle was used. The time traces were processed in the same way as the ones in the HYSCORE experiment, resulting in a 2D pattern that correlates the nuclear frequencies with the corresponding hyperfine-decoupled frequencies.

Nuclear frequency calculations and spectral simulations

The calculations of the nuclear frequencies were performed by exact diagonalization of the spin Hamiltonian taking into account the orientation selection of the experiment and were subsequently plotted to produce a HYSCORE or 2D hyperfine decoupling pattern. From the frequency calculations, the position and shape of the correlation ridges can be obtained. To make use of the information contained in the intensity of the cross-peaks, numerical simulations of HYSCORE experiments were performed in the time domain using a program written in-house [30] taking into account a four-spin system, the electron spin of the iron, and three nuclear spins of coordinating nitrogens, two from the porphyrin and one from the axial imidazole. For hyperfine decoupling experiments that use nonideal microwave pulses of variable length, another program based on the product operator formalism was developed. All pulses were treated as ideal except for the decoupling pulses, for which the propagator $\exp \left(-\mathrm{i} H T_{\mathrm{dec}}\right)$ was used, where $H$ is the Hamiltonian during microwave irradiation. The calculated time-domain data were subsequently processed in the same way as the experimental time traces.

\section{Theoretical background}

In the ferric state of LS heme systems, the five $3 d$ electrons of $\mathrm{Fe}$ (III) are located in its $t_{2 g}$ orbitals $\left(d_{z x^{\prime}}, d_{z y^{\prime}}, d_{x y}\right)$ conferring the system with $S=1 / 2$. The principal $g$ values of the paramagnetic center $\left(g_{X}=2.98, g_{Y}=2.25, g_{Z}=1.52\right)$ allow us to determine that the unpaired electron spin mainly resides in $d_{z y^{\prime}}$ with approximately $92 \%$ of the spin density $[6,20]$. This orbital is the result of rotating the standard $d_{z y}$ orbital around the heme axis $(z)^{1}$ by an angle $-\gamma$ (see Fig. 1), which, in principle, cannot be determined only with the principal values of the $g$ matrix obtained from a CW-EPR spectrum in a disordered sample $[6,18,20]$.

\footnotetext{
${ }^{1}$ Note that we denote the axes of the $g$ tensor with capital letters. The matrix elements referred to the $g$ frame have the subscript in capital letters. The lowercase subscripts refer to the molecular axes and the subscripts with primes refer to the electronic axes [20].
} 
Under the experimental conditions we work with at Qband, the spin Hamiltonian of LS heme systems is dominated by the electron Zeeman contribution, which determines the quantization axis of the electron spin. This allows one to use the high-field approximation and consider a nuclear spin Hamiltonian for each electron spin manifold, $|+\rangle$ or $|-\rangle$ [31]

$H_{n}^{ \pm}=\sum_{k=1}^{m}-g_{n, k} \beta_{n} \vec{B}_{0} \vec{I}_{k}+\left\langle\vec{S}_{\mathrm{eff}}\right\rangle^{ \pm} \hat{A}_{k} \vec{I}_{k}+\vec{I}_{k} \hat{Q}_{k} \vec{I}_{k}$

that contains the nuclear Zeeman interaction (first term), the hyperfine interaction (second term), and a third term that represents the nuclear quadrupole interaction. The last interaction is only nonzero for $I>1 / 2$ nuclei and will be considered to analyze the nuclear frequencies due to the ${ }^{14} \mathrm{~N}$ nuclei $(I=1)$ directly coordinated to the heme iron. Note that in the high-field approximation every nuclear contribution is independent and that in Eq. 1 the sum is extended to all nuclei interacting with the electron spin.

If the magnetic field is oriented along a principal direction of the $\boldsymbol{g}$ tensor (defined by the unit vector $\vec{U}$ ), then the electron spin is expected to point in the same direction. In this case and taking only nucleus $k\left(I_{k}=1\right)$ into account, we can express the Hamiltonian in Eq. 1 in matrix form as

$H_{n, k}^{ \pm}=\left(\begin{array}{ccc}-v_{k} \pm \frac{A_{U U}}{2}+\frac{1}{2} Q_{U U} & \frac{\sqrt{2}}{2}\left( \pm \frac{1}{2}\left(A_{U V}-i A_{U W}\right)+Q_{U V}-i Q_{U W}\right) & \frac{1}{2}\left(Q_{V V}-Q_{W W}\right)-i Q_{V W} \\ \frac{\sqrt{2}}{2}\left( \pm \frac{1}{2}\left(A_{U V}+i A_{U W}\right)+Q_{U V}+i Q_{U W}\right) & -Q_{U U} & \frac{\sqrt{2}}{2}\left( \pm \frac{1}{2}\left(A_{U V}-i A_{U W}\right)-Q_{U V}+i Q_{U W}\right) \\ \frac{1}{2}\left(Q_{V V}-Q_{W W}\right)+i Q_{V W} & \frac{\sqrt{2}}{2}\left( \pm \frac{1}{2}\left(A_{U V}+i A_{U W}\right)-Q_{U V}-i Q_{U W}\right) & +v_{k} \mp \frac{A_{U U}}{2}+\frac{1}{2} Q_{U U}\end{array}\right)$

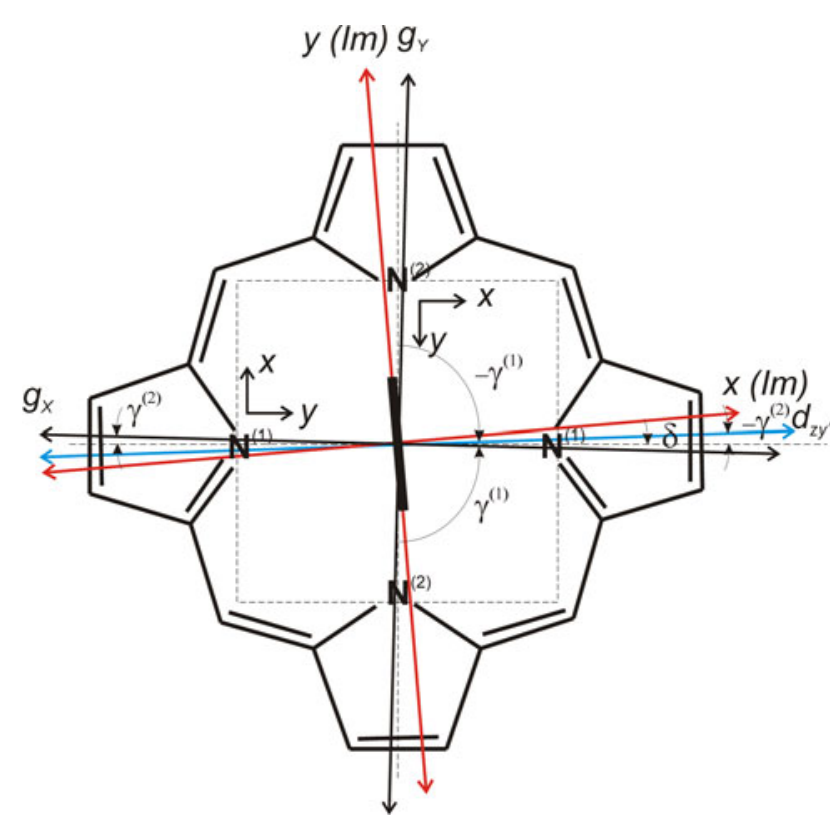

Fig. 1 The structure of the bisimidazole heme complex. The porphyrin ligand is approximately planar and the $\mathrm{Fe}(\mathrm{III})$ is approximately in the center of the ring, coordinated by its four pyrrole nitrogens. For protoporphyrin IX (PPIX), $\mathrm{R}$ is $\mathrm{CH}=\mathrm{CH}_{2}$ and for coproporphyrin IIII (CPIII), $\mathrm{R}$ is $\mathrm{CH}_{2}-\mathrm{CH}_{2}-\mathrm{COOH}$. The iron is further coordinated in the axis perpendicular to the porphyrin plane (z) by two nitrogens from two molecules of imidazole $(\mathrm{Im})$ whose plane is parallel to $z$. Here a view from the $z$-axis is shown and the plane of the imidazole ring is represented by a thick line. The direction perpendicular to the imidazole plane is represented by a red line. The axes close to the nitrogen atoms represent the frame of their nuclear quadrupole tensor and the blue line indicates the projection of the $d_{z y^{\prime}}$ orbital where $v_{k}$ is the Larmor frequency of nucleus $k$ at the given magnetic field strength and $\vec{V}$ and $\vec{W}$ define the other two principal directions of the $g$ tensor.

Under certain conditions, the exact diagonalization of the Hamiltonian expressed in Eq. 2 provides relatively simple expressions for the nuclear frequencies. For example, if the magnetic field is oriented along $Z$ (principal axis of $g$, heme normal, and the bond direction of the axial ligands), and we assume, as strongly suggested by the symmetry of the complex, that this direction is also a principal direction for the hyperfine and nuclear quadrupole interaction tensors, we find $[12,18]$

$$
\begin{aligned}
& v_{Z}^{ \pm}(\mathrm{sq} 1)=\left|\sqrt{\left( \pm \frac{1}{2} A_{Z Z}-v_{14_{\mathrm{N}}}\right)^{2}+\frac{1}{4}\left(Q_{x}-Q_{y}\right)^{2}}+\frac{3}{2} Q_{\mathrm{ZZ}}\right| \\
& v_{Z}^{ \pm}(\mathrm{sq} 2)=\left|\sqrt{\left( \pm \frac{1}{2} A_{Z Z}-v_{14_{\mathrm{N}}}\right)^{2}+\frac{1}{4}\left(Q_{x}-Q_{y}\right)^{2}}-\frac{3}{2} Q_{\mathrm{ZZ}}\right| \\
& v_{Z}^{ \pm}(\mathrm{dq})=2 \sqrt{\left( \pm \frac{1}{2} A_{Z Z}-v_{14_{\mathrm{N}}}\right)^{2}+\frac{1}{4}\left(Q_{x}-Q_{y}\right)^{2}}
\end{aligned}
$$

where $Q_{x}, Q_{y}$, and $Q_{z}\left(=Q_{z z}\right)$ are the principal values of $\boldsymbol{Q}$, sq1 and sq2 denote the two single-quantum (sq) nuclear spin transitions $\Delta m_{\mathrm{I}}= \pm 1$, and dq is the double-quantum transition, $\Delta m_{\mathrm{I}}= \pm 2$.

Even in the more general case given in Eq. 2, if the nuclear Zeeman term or the isotropic hyperfine term dominates the Hamiltonian, the matrix elements in the 


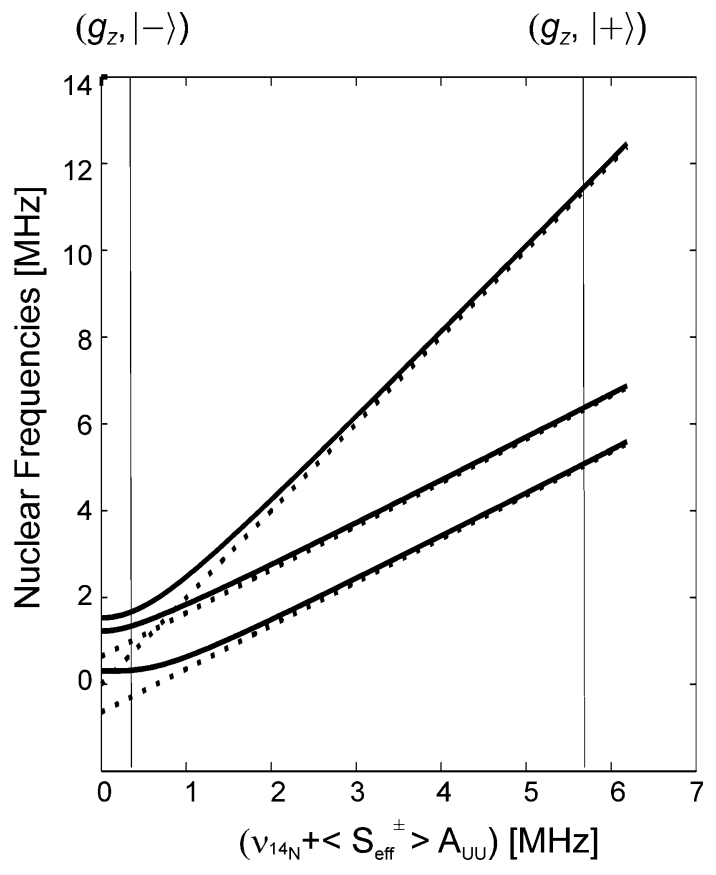

Fig. 2 Calculation of the nuclear frequencies through diagonalization of the Hamiltonian in Eq. 2. The nuclear frequencies have been plotted as a function of the secular hyperfine plus nuclear Zeeman terms. The dotted lines represent the nuclear frequencies calculated using the first-order approximation given in Eq. 4. The values of $Q_{U U}=Q_{Z Z}$ and nondiagonal hyperfine and quadrupole matrix elements of heme ${ }^{14} \mathrm{~N}$ (see Table 1) were used for the calculation. The vertical lines indicate the situation for the two different electron spin manifolds at $g_{Z}$

diagonal will determine the nuclear frequencies (see Fig. 2), which can be approximated by their first-order expressions:

$$
\begin{aligned}
& v^{ \pm}(\mathrm{sq} 1)=\left| \pm \frac{1}{2} A_{U U}-v_{k}\right|+\frac{3}{2}\left|Q_{U U}\right| \\
& v^{ \pm}(\mathrm{sq} 2)=\left| \pm \frac{1}{2} A_{U U}-v_{k}\right|-\frac{3}{2}\left|Q_{U U}\right| \\
& v^{ \pm}(\mathrm{dq})=\left| \pm A_{U U}-2 v_{k}\right|
\end{aligned}
$$

In a decoupling experiment that successfully eliminates the hyperfine interaction, the nuclear frequencies do not depend on the electron spin state because they correspond to solutions of the nuclear spin Hamiltonian (Eq.1) with $A=0$. In this case the six nuclear frequencies of Eq. 4 reduce to the three hyperfine-decoupled frequencies

$$
\begin{aligned}
v^{\mathrm{dec}}(\mathrm{sq} 1) & =\left|v_{k}\right|+\frac{3}{2}\left|Q_{U U}\right| \\
v^{\mathrm{dec}}(\mathrm{sq} 2) & =\left|v_{k}\right|-\frac{3}{2}\left|Q_{U U}\right| \\
v^{\mathrm{dec}}(\mathrm{dq}) & =\left|2 v_{k}\right|
\end{aligned}
$$

and a direct determination of the nuclear quadrupole parameters becomes possible. These methods are particularly useful when the hyperfine-decoupled spectrum is correlated to the original ESEEM spectrum in a 2D experiment. The pulse sequence used in this work employs two decoupling pulses, which allow the nuclear coherence to evolve in both electron spin manifolds, which in turn reduces the unwanted residual hyperfine splitting [23]. For sufficiently strong microwave pulses and weak hyperfine interactions, this experiment correlates the ESEEM frequencies to twice the ideally decoupled frequencies of Eq. 5.

\section{Results and discussion}

As models of LS heme proteins, two bisimidazole iron porphyrin complexes selectively labeled with ${ }^{15} \mathrm{~N}$ either in the porphyrin ring $\left[\mathrm{Fe}(\mathrm{III})-\left[{ }^{15} \mathrm{~N}\right] \mathrm{CPIII}(\mathrm{Im})_{2}\right]$ (where $\mathrm{Im}$ is imidazole) or in the imidazole [Fe(III)-PPIX $\left({ }^{15} \mathrm{~N}-\mathrm{Im}\right)_{2}$ ] were studied. Their Q-band CW-EPR spectra (not shown) display the characteristic features of LS heme systems $(S=1 / 2)$ with an anisotropic $g$ tensor $\left(g_{X}=1.52, g_{Y}=\right.$ 2.25 and $g_{Z}=2.98$ ). Such a range of $g$ values has been associated with geometries of the heme center where the two imidazole planes are approximately parallel to each other [3]. The principal value $g_{Z}=2.98$ is associated with the direction perpendicular to the heme plane; consequently the other two principal directions of the $\boldsymbol{g}$ tensor lie on the plane (see Fig. 1). Selective labeling of the complexes with ${ }^{15} \mathrm{~N}$ allows the hyperfine couplings of the heme and imidazole nitrogen nuclei to be studied separately.

\section{Imidazole nitrogens}

The electron spin echo obtained at $g=g_{Z}$ for the complex $\mathrm{Fe}(\mathrm{III})-\left[{ }^{15} \mathrm{~N}\right] \mathrm{CPIII}(\mathrm{Im})_{2}$, labeled with ${ }^{15} \mathrm{~N}$ in the porphyrin ring, is very strongly modulated. The HYSCORE spectrum at this observer position (Fig. 3a) shows a pair of strong peaks in the $(-,+)$ quadrant centered at around $4 \mathrm{MHz}$ whose abscissa and ordinate differ by $2 v_{15_{\mathrm{N}}}=7.17 \mathrm{MHz}$. Consequently, these peaks are assigned to the nitrogen nuclei of the porphyrin. Additionally, strong correlation peaks that can be attributed to ${ }^{14} \mathrm{~N}$ are found in both quadrants (the exact positions of the peaks are collected in Table S1). These peaks, which are very close to the abscissa or ordinate, are elongated along the direction of the axes to which they are close. This means that the distribution of nuclear frequencies is broader in the electron spin manifold where the hyperfine and nuclear Zeeman frequencies add together $(|+\rangle$, as the sign of the hyperfine coupling is known to be negative [19]) than in $|-\rangle$. Note that all the nuclear frequencies can be understood with one ${ }^{14} \mathrm{~N}$ nucleus and one ${ }^{15} \mathrm{~N}$ nucleus, which evidences that for this observer position the two nitrogen nuclei from the imidazole are magnetically equivalent, as well as the four heme nitrogens, reflecting the symmetry of the complex. 
Fig. 3 a Hyperfine sublevel correlation spectroscopy (HYSCORE) spectrum of the complex $\mathrm{Fe}(\mathrm{III})-\left[{ }^{15} \mathrm{~N}\right] \mathrm{CPIII}(\mathrm{Im})_{2}$, taken at the $g=g_{Z}$ observer position. $\boldsymbol{B}=830 \mathrm{mT}, \tau=208 \mathrm{~ns}$, $T=6 \mathrm{~K}$. b Simulation with the parameters collected in Table 1

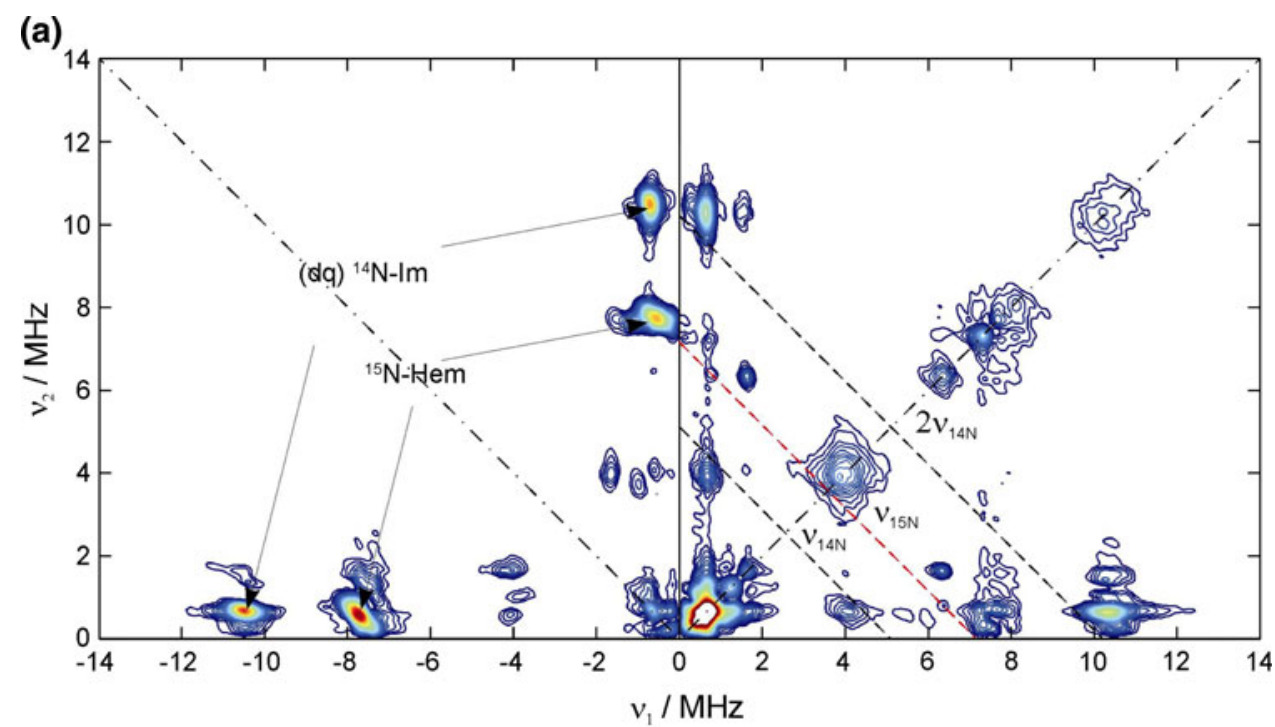

(b)

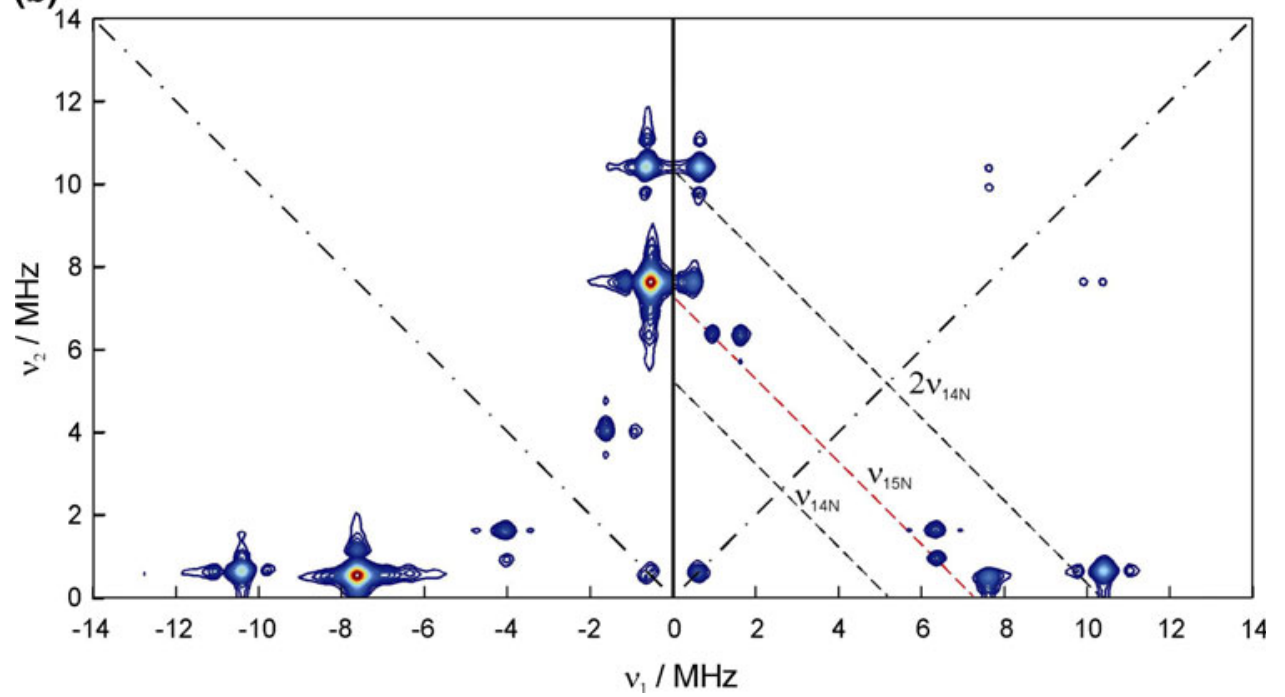

Using the ${ }^{14} \mathrm{~N}$ frequencies of the $|+\rangle$ manifold determined from the HYSCORE spectrum, the first-order approximation provides the values $\left|A_{Z Z}\right|=5.2 \mathrm{MHz}$ and $\left|Q_{Z Z}\right|=0.85 \mathrm{MHz}$. Note that $A_{Z Z}$ is approximately twice $v_{14_{\mathrm{N}}}$, which means that the secular parts of the hyperfine and the nuclear Zeeman interactions cancel each other in the $|-\rangle$ electron spin manifold. Therefore, for this spin manifold the first-order formulae of Eq. 4 are not valid and exact diagonalization of Eq. 2 is needed. From Eq. 3 we get

$\left|v_{Z}^{+}(\mathrm{dq})^{2}-v_{Z}^{-}(\mathrm{dq})^{2}\right|=\left|8 A_{Z Z} v_{14_{N}}\right|$

and

$v_{Z}^{+}(\mathrm{dq})^{2}+v_{Z}^{-}(\mathrm{dq})^{2}=8 v_{14_{\mathrm{N}}}^{2}+2 A_{Z Z}^{2}+2\left(Q_{x}-Q_{y}\right)^{2}$

Using the dq frequencies obtained from the dq correlation peaks $(10.38,0.63) \mathrm{MHz}$ and $(0.63,10.38) \mathrm{MHz}$, Eq. 6a gives $\left|A_{Z Z}\right|=5.25 \mathrm{MHz}$. In addition, by setting this value in Eq. 6b, we determine $\left|Q_{x}-Q_{y}\right|=0.64 \mathrm{MHz}$. Note that the only assumption made here is that the direction of $g_{Z}$ coincides with one principal axis of the nuclear quadrupole tensor. Writing this tensor in the standard diagonal form $[-K(1-\eta),-K(1+\eta), 2 K]$, there are three possible ways to express $Q_{x}-Q_{y}$ as a function of the parameters $K$ and $\eta: Q_{x}-Q_{y}=2 K \eta \quad Q_{x}-Q_{y}=K(3-\eta)$, and $Q_{x}-Q_{y}=K(3+\eta)$. From the sq correlation peaks, which are very sensitive to the nuclear quadrupole parameters, we can safely exclude the last two cases and conclude that $K \eta=0.32 \mathrm{MHz}$. This constraint allows for an accurate determination of $K=-0.42 \mathrm{MHz}$ (sign obtained from [32]) and $\eta=0.75$ through the analysis of the peak positions in the HYSCORE spectrum. Indeed, also the complete simulation (including the intensities) illustrated in Fig. 3b reproduces well most of the features of the correlation pattern.

This situation of exact cancellation allows us to obtain the principal values $Q_{x}, Q_{y}$, and $Q_{z}$ from the nuclear 
Table 1 Summary of hyperfine and nuclear quadrupole interaction parameters for the directly coordinated ${ }^{14} \mathrm{~N}$ of the heme and axial imidazole

\begin{tabular}{|c|c|c|c|}
\hline & \multirow[t]{2}{*}{${ }^{14} \mathrm{~N}$-imidazole } & \multicolumn{2}{|l|}{${ }^{14} \mathrm{~N}$-porphyrin ${ }^{\mathrm{a}}$} \\
\hline & & (2) & (1) \\
\hline$A_{X X}{ }^{\mathrm{b}}$ & $-5.5 \pm 0.1$ & $-4.9 \pm 0.1$ & $-4.5 \pm 0.1$ \\
\hline$A_{X Y}$ & $0.1 \pm 0.1$ & $0.0 \pm 0.1$ & $0.0 \pm 0.1$ \\
\hline$A_{Y Y}$ & $-6.2 \pm 0.1$ & $-4.8 \pm 0.1$ & $-4.6 \pm 0.1$ \\
\hline$A_{Z Z}$ & $-5.2 \pm 0.1$ & $-5.9 \pm 0.1$ & $-5.9 \pm 0.1$ \\
\hline$A_{Z X}$ & $0.0 \pm 0.1$ & $0.3 \pm 0.1$ & $0.0 \pm 0.6$ \\
\hline$A_{Z Y}$ & $0.0 \pm 0.1$ & $0.0 \pm 0.6$ & $0.3 \pm 0.1$ \\
\hline$Q_{x}^{\mathrm{b}}$ & $0.11 \pm 0.05$ & $0.93 \pm 0.05$ & $0.93 \pm 0.05$ \\
\hline$Q_{y}$ & $0.74 \pm 0.05$ & $-0.51 \pm 0.05$ & $-0.51 \pm 0.05$ \\
\hline$Q_{z}$ & $-0.85 \pm 0.05$ & $-0.42 \pm 0.05$ & $-0.42 \pm 0.05$ \\
\hline Angle $\left({ }^{\circ}\right)$ & $\delta=-5 \pm 5$ & $\gamma^{(2)}=0 \pm 5$ & $\gamma^{(1)}=90 \pm 5$ \\
\hline$\beta\left(^{\circ}\right)$ & $0 \pm 5$ & $+10 \pm 5$ & $-10 \pm 5$ \\
\hline
\end{tabular}

The elements of the hyperfine matrix are given in megahertz in the frame of the $g$-tensor principal axes. For the nuclear quadrupole tensors the principal values are given. The tilt angle $\beta$ connecting the $z$ principal axis of $\boldsymbol{Q}$ with the one of $g$ in a passive rotation is given in degrees. The relative orientation of the $\boldsymbol{g}$ and $\boldsymbol{Q}$ tensors in the heme plane is given in degrees by the angles $\delta$ and $\gamma$ (see Fig. 1)

a The two columns correspond to the two sets of ${ }^{14} \mathrm{~N}$-heme nuclei (see the text)

b The signs of the hyperfine components have been assigned according to [19] and those of the quadrupole according to [32]. Hyperfine values obtained from X-band data [18], in megahertz: ${ }^{14} \mathrm{~N}$-imidazole $\left(\left|A_{X X}\right|=5.6 \pm 0.2,\left|A_{X Y}\right|<0.7,\left|A_{Y Y}\right|=6.2 \pm 0.3,\left|A_{X Z}\right|=\left|A_{Y Z}\right|=0,\left|A_{Z Z}\right|=\right.$ $5.1 \pm 0.5), \mathrm{N}-\mathrm{Hem}\left(\left|A_{X X}\right|=4.7 \pm 0.2,\left|A_{X Y}\right|<1.3,\left|A_{Y Y}\right|=4.9 \pm 0.4,\left|A_{X Z}\right|=\left|A_{Y Z}\right|=0,\left|A_{Z Z}\right|=5.8 \pm 0.6\right)$

frequencies with a relatively high precision $\left(Q_{x}=\right.$ $-0.11 \mathrm{MHz}, Q_{y}=-0.74 \mathrm{MHz}$, and $Q_{z}=0.85 \mathrm{MHz}$, see the summary of all parameters in Table 1). The principal axes of $\boldsymbol{Q}$ follow the directions of symmetry of the imidazole ring [perpendicular to the imidazole plane, Fe(III)$\mathrm{N}(\mathrm{Im})$ direction, and its vector product]. Indeed, from the nuclear frequencies of the $|+\rangle$ manifold, we found $\left|Q_{Z Z}\right|=$ $0.85 \mathrm{MHz}$, one of the principal values of the tensor, confirming the fact that the $Z$-axis [Fe(III)-N(Im) direction] is also a principal axis of $\boldsymbol{Q}$.

Simulations of the HYSCORE spectrum using $A_{Z X}=0$ and $A_{Z Y}=0$ yield satisfactory results (see Fig. $3 \mathrm{~b}$ ) so we can conclude that $Z$ is also a principal axis of $\boldsymbol{A}$, in agreement with the fact that the heme normal is one of the electronic axes of the orbitals among which the unpaired electron is distributed.

The principal direction corresponding to observer position $g_{X}$ is in the heme plane, but its exact orientation is a priori unknown. The nuclear frequencies will depend on the hyperfine as well as on the nuclear quadrupole interaction, for none of which is $\mathrm{X}$, in principle, a principal axis. In this situation there are more parameters to be adjusted (namely, principal values of $\boldsymbol{Q}$ and $\boldsymbol{A}$ and orientation of the axes in the heme plane) and therefore it is more complicated to determine them unambiguously. Consequently, it would be extremely useful to reduce or separate these contributions. One possibility in this direction is to use ${ }^{15} \mathrm{~N}$ labeling in the axial imidazole rings, for which $I=1 / 2$ and there is no nuclear quadrupole interaction (see the following section). The other one, more convenient when dealing with proteins where isotope labeling is much more demanding, is to suppress the hyperfine coupling by performing hyperfine-decoupling experiments. This allows for a more direct and accurate determination of the nuclear quadrupole parameters, which contain information regarding the orientation of axial imidazole rings with respect to the $g$ frame.

Recently, using a modified version of the hyperfinedecoupled DEFENCE sequence [24], we showed that under favorable conditions (weak coupling case) the residual hyperfine splitting can be completely cancelled so that the experimental decoupled frequencies are determined only by the nuclear Zeeman and quadrupole interactions [23]. Figure $4 \mathrm{~b}$ shows the decoupling spectrum at this observer position. The abscissa of the peaks in the spectrum corresponds to the nuclear frequencies ( $v_{\text {ESEEM }}$ ) and the ordinate to twice the decoupled frequencies, that is, the nuclear frequencies which are free from hyperfine coupling, $A=0$. The magnetic field at this observer position is $1,650 \mathrm{mT}$ and thus the nuclear Zeeman terms of both ${ }^{14} \mathrm{~N}$ and ${ }^{15} \mathrm{~N}$ dominate the nuclear spin Hamiltonians, so the first-order hyperfine-decoupled frequencies can be approximated by the formulas of Eq. 5. In Fig. 4b one can observe signals at $2 v_{\mathrm{dec}}=2 v_{15_{\mathrm{N}}}$ correlated to two ESEEM frequencies which are assigned to the heme ${ }^{15} \mathrm{~N}$ nuclei. Although no signals can be detected around $2 v_{\text {dec }}=4 v_{14_{\mathrm{N}}}$, one can easily distinguish two peaks at $2 v_{\mathrm{dec}} \sim 2 v_{14_{\mathrm{N}}}$ (see Table S2 for the exact peak positions) elongated along the 
Fig. 4 a HYSCORE spectrum of $\mathrm{Fe}(\mathrm{III})-\left[{ }^{15} \mathrm{~N}\right] \mathrm{CPIII}(\mathrm{Im})_{2}$ taken at the observer position $g_{X}$ $(\boldsymbol{B}=1,690 \mathrm{mT})$; the spectrum displayed is the sum of the spectra recorded with $\tau=170$, 96, and 208 ns. b Hyperfine decoupling spectrum of the same sample also taken at $g_{X}$ $(\boldsymbol{B}=1,650 \mathrm{mT}) . T=6 \mathrm{~K} . \mathbf{c}, \mathbf{d}$ Simulations of $\mathbf{a}$ and $\mathbf{b}$ with the parameters collected in Table 1 (a)

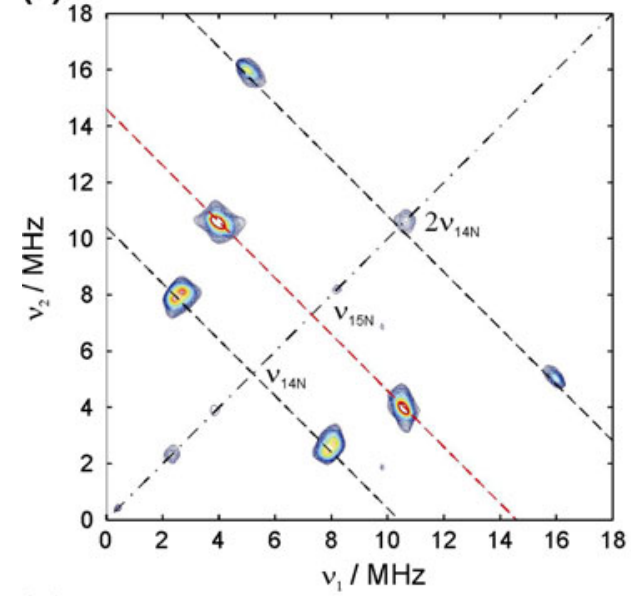

(b)

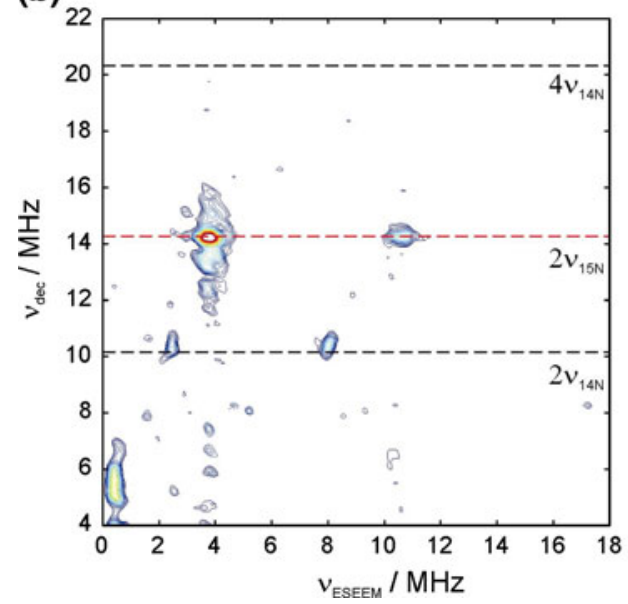

(c)

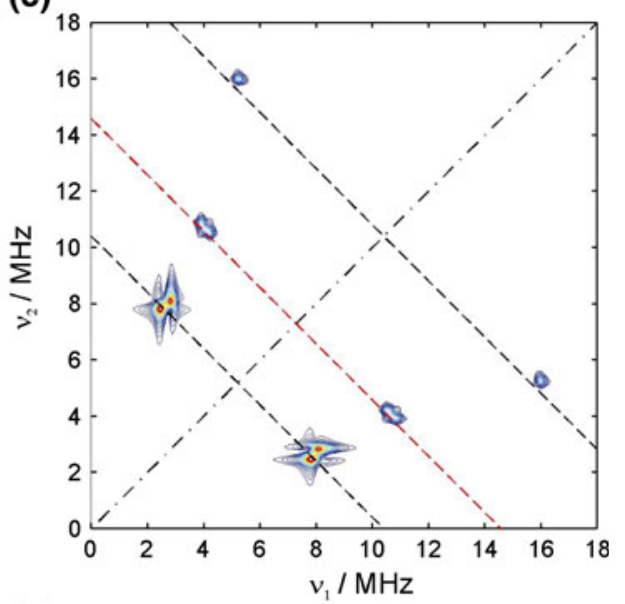

(d)

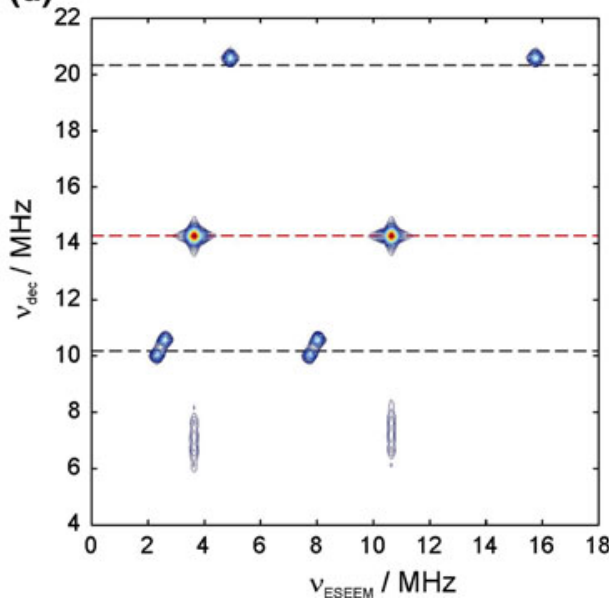

decoupling dimension that one can assign to one ${ }^{14} \mathrm{~N}$ nucleus with $Q_{X X}$ very close to zero.

Now it has been established that the $Z$-axis is also a principal axis of $\boldsymbol{Q}$, the matrices representing tensor $\boldsymbol{Q}$ in its own principal axes system $x y z$ and in the $g$-frame $X Y Z$ are related by a rotation in the heme plane (of a generic angle $\delta$ ). Then, the matrix element $Q_{X X}$ is expressed as a function of $Q_{x}$ and $Q_{y}$ :

$Q_{X X}=Q_{x} \cos ^{2} \delta+Q_{y} \sin ^{2} \delta$

From the decoupling experiment we can estimate that $\left|Q_{X X}\right|=0.06 \mathrm{MHz}$. Since the principal values $Q_{x}$ and $Q_{y}$, were previously determined from the analysis of the HYSCORE spectrum obtained at $g=g_{Z}$, the angle $\delta$ between the $x$ - and $X$-axes can be determined easily using Eq. 7. Spectral simulations of the line intensities were performed; the results shown in Fig. $4 \mathrm{~d}$ are the most satisfactory, corresponding to $\delta=-5^{\circ} \pm 5^{\circ}$. The fact that we can distinguish only one imidazole nucleus at this observer position indicates that the planes of the two axial imidazole ligands are parallel (within an angle of $5^{\circ}$ )
The HYSCORE spectrum recorded at the same observer position is shown in Fig 4a; the negative quadrant is empty, so only the positive quadrant is shown. All of the ESEEM frequencies coincide with the ones observed in the hyperfine decoupling experiment. The spectrum is dominated by the correlations of ${ }^{15} \mathrm{~N}$ from the porphyrin, but one can clearly distinguish also two pairs of (sq, sq) correlations very close to each other and a (dq, dq) peak from the imidazole nitrogens at higher frequencies (see Table S3 for the exact peak positions). Simulations of this spectrum allowed us determine $A_{X X}$ and $A_{X Y}$ as they are presented in Table 1.

The determination of the coupling tensors was completed by analysis and simulation of the spectra at observer position $g_{Y}$. Although this is not a single-crystal spectrum and in general it is more difficult to unambiguously obtain precise information about tensor orientations, this information was already obtained from the other experiments and the spectra at $g_{Y}$ were only used to find the coupling parameters along axis $Y$ (spectrum and simulations in the electronic supplementary material). 
The above accurate determination of the nuclear quadrupole parameters can be further used to reveal the orientation of the imidazole plane relative to the principal axes system of $\boldsymbol{g}$, provided that the orientation of $\boldsymbol{Q}$ in the imidazole plane is known. Early nuclear quadrupole resonance studies on imidazole-metal complexes of $\mathrm{Cd}(\mathrm{II})$ and $\mathrm{Zn}$ (II) [33] showed that for the imino nitrogen directly coordinated to the metal the larger matrix element $Q_{z}=2 \mathrm{~K}$ lies along the $\mathrm{Fe}-\mathrm{N}_{\operatorname{Im}}(Z)$ direction (which is in line with our previous assignment), whereas the smaller element $Q_{x}=-K(1-\eta)$ is oriented perpendicular to the imidazole plane. Since the HYSCORE and hyperfine decoupling experiments performed at $g_{X}$ showed that the quadrupole splitting is very small at this observer position, one can conclude that the imidazole plane is approximately $\left(-5^{\circ}\right.$ according to decoupling simulations) perpendicular to the $X$-axis as illustrated in Fig. 1.

The hyperfine coupling constants that we have found here (collected in Table S3) are within the error limits of the ones found in similar complexes $[13,18]$ using $X$-band data and they have better precision. To interpret the hyperfine tensor obtained, one has to take into account the nature of the ground state and the effect of the large anisotropy of the $g$ tensor which result in a nonisotropic Fermi contact and nonsymmetric/nontraceless dipole contributions. According to [20], the trace of the Fermi contribution is $2.68 a_{\text {iso }} \mathrm{MHz}$. If we consider that the electron density located on the iron is far from the axial nitrogen, we can easily estimate its contribution to the hyperfine interaction with the point-dipole expression $\hat{A}_{\mathrm{dd}}=\frac{1}{g_{e}} \hat{g} \hat{T}$, where $\hat{T}$ is the dipole coupling tensor [28]. Computing the trace of the experimental hyperfine tensor, we found a dominating isotropic contribution of $-6.6 \mathrm{MHz}$. The negative sign of the isotropic contribution evidences spin polarization of the $s$ orbitals of the nitrogen by the iron, in agreement with what has been obtained through density functional theory calculations [34]. However, to fully account for the hyperfine tensor found experimentally, one needs to consider an extra, traceless contribution $\left(A^{\prime}\right.$ in Eq. 8) closely axial along $Z,\left(A_{Z}^{\prime}=-0.9 \mathrm{MHz}\right)$, which most probably reflects the polarization of the $\mathrm{Fe}-\mathrm{N} \sigma$ bond predicted by density functional theory calculations:

$A=A_{F}+A_{\mathrm{dd}}+A^{\prime}$

Heme nitrogens

Figure 5a displays the Q-band HYSCORE spectrum of Fe(III)-PPIX $\left({ }^{15} \mathrm{~N}-\mathrm{Im}\right)_{2}$ taken at observer position $g_{Z}$. In the $(-,+)$ quadrant several correlation peaks due to ${ }^{14} \mathrm{~N}$ are visible (see the assignment in Table S1). These peaks are located close to the axes, as was the case for the imidazole nitrogens at the same field position, which indicates that they are also close to the exact cancellation condition. Indeed, some of the ${ }^{14} \mathrm{~N}$ features are replicated in the $(+,+)$ quadrant. The weak cross-peaks at $(7.3,0.3)$ $\mathrm{MHz}$ fall in the second antidiagonal and are assigned to the imidazole ${ }^{15} \mathrm{~N}$ directly coordinating the iron. Their low intensity is due to the fact that $Z$ is a principal axis of $\boldsymbol{A}$ and consequently there is little branching.

The strong correlation peaks that appear at lower frequencies combine ${ }^{15} \mathrm{~N}$ and ${ }^{14} \mathrm{~N}$ nuclear frequencies (see Table S1). As with ${ }^{14} \mathrm{~N}$ imidazole, incomplete inversion by $\pi$ pulse leads to some peaks along the diagonal, signaling also some of the ${ }^{14} \mathrm{~N}$ nuclear frequencies. All the nuclear frequencies at this field position can be explained by two equivalent ${ }^{14} \mathrm{~N}$ nuclei and one ${ }^{15} \mathrm{~N}$ nucleus, so the possible differences among the four heme ${ }^{14} \mathrm{~N}$ and the two axial ${ }^{15} \mathrm{~N}$ are small and not resolved.

In the $|+\rangle$ manifold one can safely apply the first-order expressions of Eq. 4 to find $\left|Q_{Z z}\right|=0.42 \pm 0.05 \mathrm{MHz}$ and $\left|A_{z Z}\right|=5.9 \mathrm{MHz}$ for heme nitrogens. This hyperfine coupling is in good agreement with the scaled value of $\frac{g_{14} \mathrm{~N}}{g_{15 \mathrm{~N}}} A^{15_{\mathrm{N}}}=-6.0 \mathrm{MHZ}$, where $\mathrm{A}^{15_{\mathrm{N}}}$ is the ${ }^{15} \mathrm{~N}$-heme hyperfine coupling obtained from the experiment reported in Fig. 3. The hyperfine coupling does not fulfil precisely the exact cancellation condition for the $|-\rangle$ manifold and leaves a diagonal term $\left(-A_{Z Z} / 2-v_{14 \mathrm{~N}} \sim 0.3 \mathrm{MHz}\right)$ that is probably comparable to the off-diagonal nuclear quadrupole terms (see Fig. 2). We use then the third expression in Eq. 3, together with the value of $\left|Q_{z z}\right|$ to find $\left|Q_{x}\right|=$ $0.97 \mathrm{MHz}$ and $\left|Q_{y}\right|=0.54 \mathrm{MHz}$. Note that the difference between the two sq transitions, which according to Eq. 3 should be $3 Q_{\mathrm{ZZ}}$, is not the same in both electron spin manifolds (see Table S1). This discrepancy must come from nondiagonal terms that were neglected when deriving the expressions, that is, nonexact collinearity of the three tensors $\boldsymbol{g}, \boldsymbol{A}$, and $\boldsymbol{Q}$ along the $z$-axis. These extra terms influence more the nuclear frequencies in the $|-\rangle$ spin manifold because of the weaker diagonal terms.

Simulations of the spectrum confirm this point, since, although the peak positions can be approximately reproduced considering the heme normal is a principal axis of all tensors and the values for $A_{Z Z}$ and nuclear quadrupole tensor principal values given above, this is not the case for the relative intensities of the peaks. On the other hand, if we consider $\left|A_{Z X}^{(2)}\right|=0.3 \mathrm{MHz}$ and the other values summarized in Table 1, a satisfactory agreement with the experimental finding can be obtained (see Fig. 5b). The simulations are not so sensitive to $\left|A_{Z Y}^{(2)}\right|$, in the simulation shown we have considered $\left|A_{Z Y}^{(2)}\right|=0$ but the error is considerably larger in the estimation of this parameter. The inclusion of these nondiagonal terms results in a deviation between the principal axes of the $\boldsymbol{g}$ and $\boldsymbol{A}$ tensors $(\beta)$ of about $10^{\circ}$ which most probably reflects a small departure of the heme plane from planarity. Variation of the nuclear quadrupole parameters in the frequency calculations and 
Fig. 5 a HYSCORE spectrum of Fe(III)-PPIX $\left({ }^{15} \mathrm{~N}-\mathrm{Im}\right)_{2}$ taken at the observer position $g_{\mathrm{Z}}$. $\boldsymbol{B}=869.3 \mathrm{mT}, T=6 \mathrm{~K}$, $\tau=144$ ns. b Simulation of the

${ }^{14} \mathrm{~N}$ features in a using the parameters given for heme nitrogen nuclei in Table 1

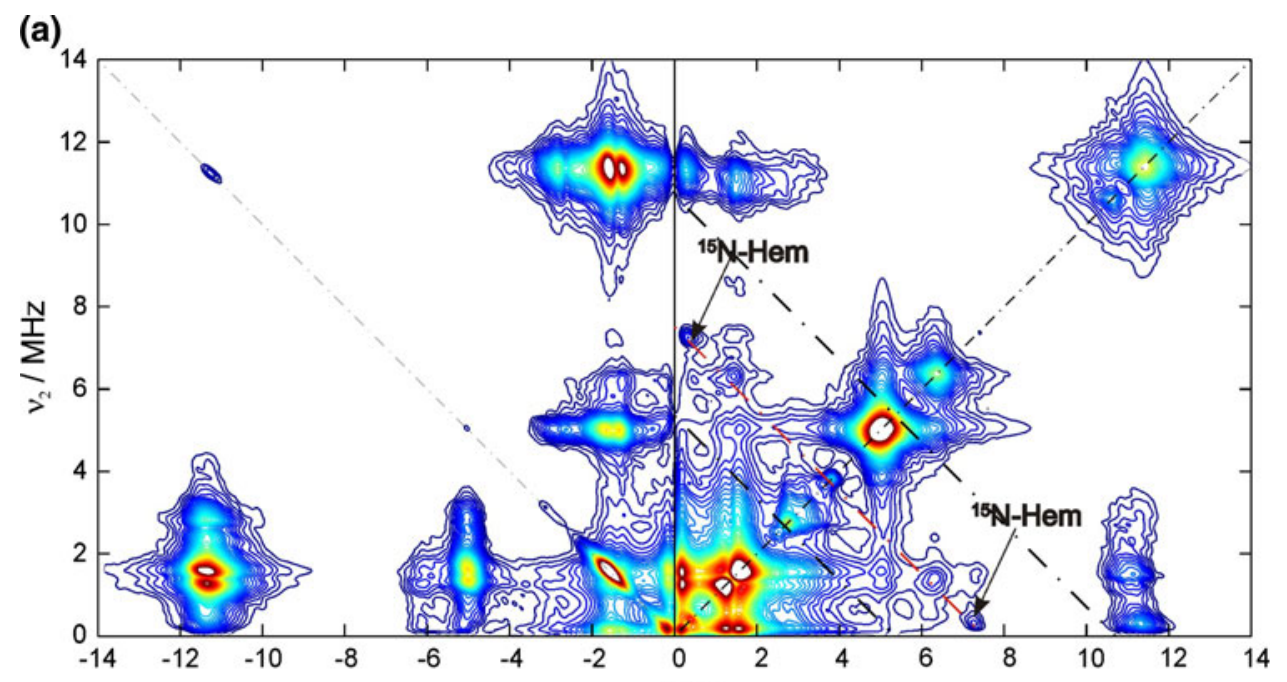

(b) $\quad v_{1} / \mathrm{MHz}$

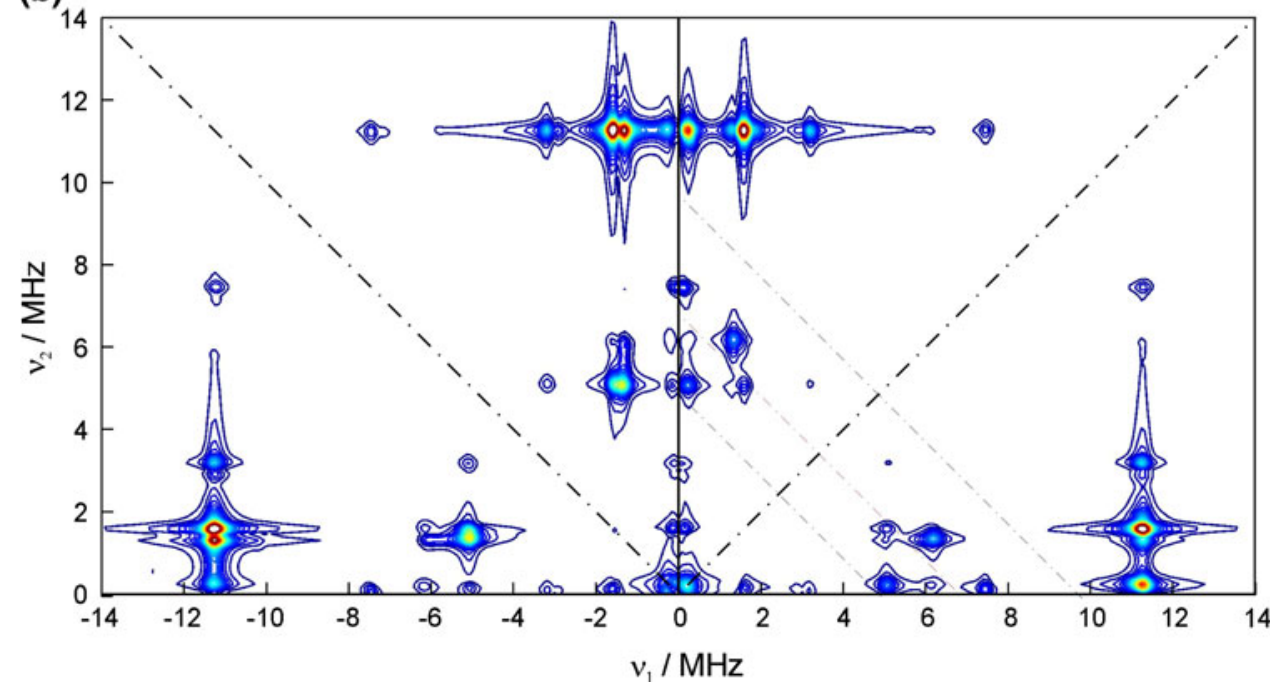

spectral simulations allowed refinement of the $Q_{x}$ and $Q_{y}$ principal values. Nonzero elements $Q_{X Z}$ and $Q_{Y Z}$ were also added to reflect the deformation of the porphyrin ring but did not have much effect in the simulation.

The results obtained at the other single-crystal observer position $\left(g_{X}\right)$ are displayed in Fig. 6. Since the heme nitrogens are also in the weak coupling regime at this field position, the same strategy as for the imidazole nitrogens can be used. The decoupled frequencies were obtained to find out the orientation of the $X$-axis $(g)$ in the molecular frame (heme) and then HYSCORE experiments were performed to obtain the hyperfine parameters. In the hyperfine decoupling spectrum (Fig. 6b) we find again two intense peaks situated along $2 v_{15_{\mathrm{N}}}$ in the decoupling dimension which are assigned to the axially coordinating nitrogen of the imidazole. Well above the noise level two couples of peaks with the same ordinate can be distinguished at around $8 \mathrm{MHz}$ in the decoupling dimension (see Table S2 for the exact peak positions), both at the lower side of the dotted line at $2 v_{14_{\mathrm{N}}}$. They are assigned to two different ${ }^{14} \mathrm{~N}$ nuclei, to be exact, to the two nonequivalent pairs, (1) and (2), of diagonally opposed ${ }^{14} \mathrm{~N}$-heme nuclei. According to first order, the difference between the peaks in the spectrum and $2 v_{14_{\mathrm{N}}}$ is equal to $3 Q_{X X}$ and there should be another pair of lines above $2 v_{14_{\mathrm{N}}}$, at the same distance. We find this second, weaker pair of lines for nitrogens (1) but not for nitrogens (2).

Again, considering that the $Q_{X X}$ matrix element can be expressed as a function of the principal values of the nuclear quadrupole tensor in the heme plane, $Q_{x}$ and $Q_{y}$ (associated with the molecular axes), and the angle $\gamma$ between the $X$-axis and molecular axes $x$ and $y$, we can write

$\left|Q_{X X}^{(1)}\right|=Q_{x} \cos ^{2} \gamma^{(1)}+Q_{y} \sin ^{2} \gamma^{(1)}$
$\left|Q_{X X}^{(2)}\right|=Q_{x} \cos ^{2} \gamma^{(2)}+Q_{y} \sin ^{2} \gamma^{(2)}$

From the experimental decoupled nuclear frequencies we can directly find that $\left|Q_{X X}^{(1)}\right|=1.45 / 3=0.48 \mathrm{MHz}$ and 
Fig. 6 a HYSCORE spectrum of $\mathrm{Fe}(\mathrm{III})-\mathrm{PPIX}\left({ }^{15} \mathrm{~N}-\mathrm{Im}\right)_{2}$ taken at the observer position $g_{X}$; the spectrum displayed is the sum of the spectra recorded with $\tau=96$ and $208 \mathrm{~ns}$. The antidiagonal lines cross the diagonal at $v_{14_{\mathrm{N}}}, v_{15_{\mathrm{N}}}$, and $2 v_{14_{\mathrm{N}}}$. b Hyperfine decoupling experiment of the same complex also at the $g_{X}$ observer position $\tau=96 \mathrm{~ns}$. The abscissa corresponds to the electron spin echo envelope modulation frequencies and the ordinate corresponds to the decoupled nuclear frequencies. For $\mathbf{a}$ and b, $\boldsymbol{B}=1,650 \mathrm{mT}, T=6 \mathrm{~K} . \mathbf{c}, \mathbf{d}$ Simulations of $\mathbf{a}$ and $\mathbf{b}$ with the parameters collected in Table 1 (a)

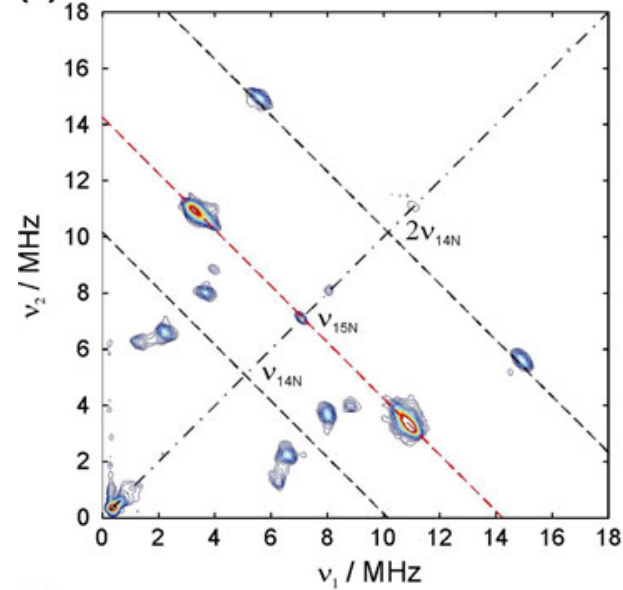

(b)

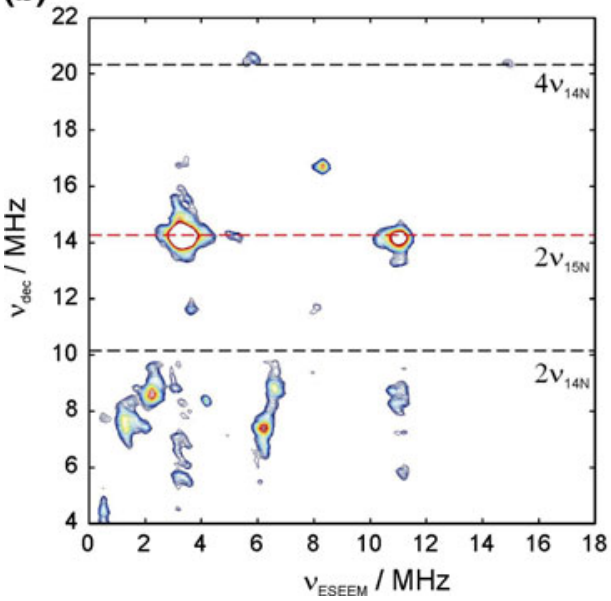

(c)

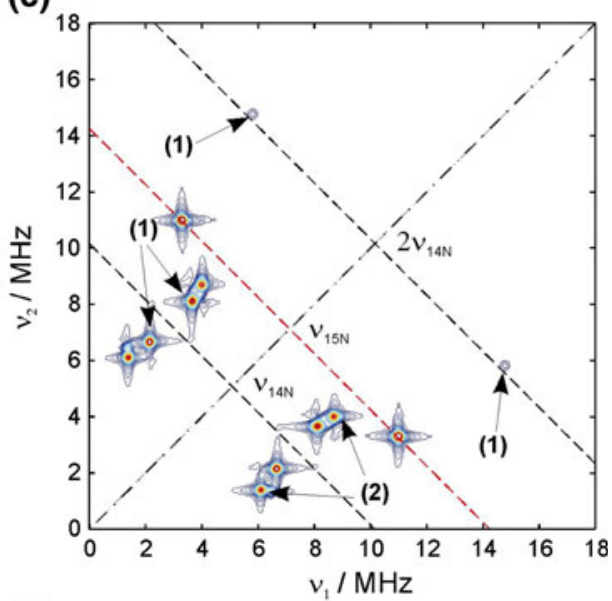

(d)

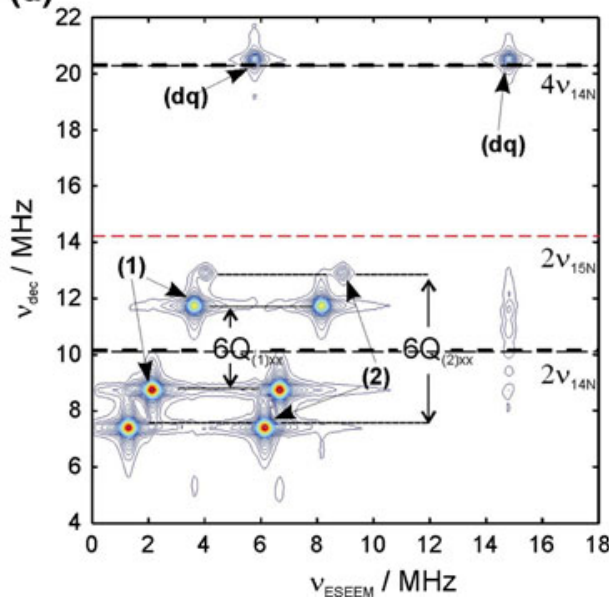

$\left|Q_{X X}^{(2)}\right|=2.76 / 3=0.92 \mathrm{MHz}$. Setting these values in Eq. 9, we find $\left|\gamma^{(1)}\right|=86^{\circ}$ and $\left|\gamma^{(2)}\right|=0^{\circ}$. Simulations of the decoupling experiment yielded the best results for $\left|\gamma^{(1)}\right|=$ $90^{\circ}$ and $\left|\gamma^{(2)}\right|=0^{\circ}$, so we can conclude that, if any, the rotation angle between the $\mathrm{g}$-tensor and the $\mathrm{N}-\mathrm{Fe}-\mathrm{N}$ directions is very small. Note that the intensities of the decoupling simulations are very sensitive to the angles $\gamma$ (and their sign), as well as the tilt angles $(\beta)$ between $Q_{x}$ and $g_{Z}$. That $\gamma^{(1)}$ and $\gamma^{(2)}$ are found from independent experimental evidence and $\gamma^{(1)}+\gamma^{(2)}=90^{\circ}$ fulfils an internal consistency test, as we know the two pairs of nitrogens in the porphyrin ring are arranged perpendicularly to each other.

The HYSCORE spectrum recorded at $g_{X}$, shown in Fig. 6a, is again dominated by the correlations of ${ }^{15} \mathrm{~N}$ from the axial imidazole rings, but one can clearly also distinguish (sq, sq) correlations due to two nonequivalent pairs of ${ }^{14} \mathrm{~N}$. Also a (dq, dq) peak from the heme nitrogen is visible at higher frequencies (see Table S3 for the exact peak positions). All of the ESEEM frequencies coincide with the ones observed in the hyperfine decoupling experiment and, as found for the imidazole nuclear frequencies, once the parameters of the nuclear quadrupole coupling are known, the determination of the parameters of the hyperfine coupling through simulation of the HYSCORE spectra is much simpler. The two pairs of heme nitrogens were found to have similar hyperfine couplings with a somewhat different $A_{X X}$ (see Table 1) and also negligible $A_{X Y}$ coupling. This, together with the information retrieved about the nuclear quadrupole interaction, means that the $X$-axis of the complex is very close to one of the $\boldsymbol{Q}$ and $\boldsymbol{A}$ principal axes of the heme nitrogens.

It should be noted that, quite the opposite to what was found at X-band [18], the experimental results presented here for this observer position allow us to obtain the hyperfine and nuclear quadrupole couplings unambiguously. This is partially due to the fact that the quadrupole coupling has been separated from the hyperfine coupling by hyperfine decoupling experiments. In addition, the better orientation selection achieved at Q-band frequencies and the increased nuclear Zeeman contribution that sets the Hamiltonian away from the exact cancellation condition result in well-defined and separated peaks that can be 
assigned and interpreted easily. On the other hand, at Xband the correlation features were extended and crossing ridges were interpreted as $\gamma$ being close to $45^{\circ}$, as the nuclear frequencies then depend strongly on the orientation. In reality, we have found here that $\gamma$ is close to zero and the ridges appear as a consequence of the small diagonal terms in the Hamiltonian for the spin manifold $|-\rangle$ which make the nuclear frequencies more sensitive to nonsecular terms. These terms depend strongly on small variations of the orientation of the center and therefore produce ridges when combined with a large $g$ strain and poorer orientation selection.

The analysis and simulation of HYSCORE spectra at observer position $g_{Y}$ (see the electronic supplementary material) allowed the completion of Table 1.

The hyperfine tensor of heme nitrogens also shows an isotropic dominating contribution of about -5.8 and $-5.4 \mathrm{MHz}$ for the pair of nitrogens aligned along the $y$ and $x$ molecular axes, respectively. This difference is not significant with respect to the error margin but it could still reflect a small asymmetry in the heme plane. If the contribution to the spin density on the iron is, again, approximated by the point-dipole formula above, an additional contribution is needed to account for the experimental hyperfine couplings which is not very far from axial with the main axis close to the $\mathrm{N}-\mathrm{Fe}$ bond $\left(A_{Y}^{\prime}=-1.3 \mathrm{MHz}\right.$ and $A_{X}^{\prime}=-1.1 \mathrm{MHz}$ for the pair of nitrogens aligned along the $Y$ - and $X$-axes, respectively). Again, we interpret this contribution as coming from the polarization of the $\sigma$ bonds with the iron [34]. Moreover, the finding that $\boldsymbol{A}$ is not exactly aligned with $\boldsymbol{g}$ (which is aligned with the $\mathrm{Im}-\mathrm{Fe}$ direction) reflects slight ruffling of the porphyrin ring, probably close to the $10^{\circ}$ estimated for the deviation of the hyperfine coupling from the $Z$-axis. X-ray structures of a very similar complex reported a ruffling angle close to $8^{\circ}$ [35].

Since it is known that the direction corresponding to the larger quadrupole principal value $\left(Q_{x}\right)$ lies on the heme plane perpendicular to the $\mathrm{N}-\mathrm{Fe}$ bond [32], we can display $g_{X}$ close to the direction $\mathrm{N}^{(1)}-\mathrm{Fe}-\mathrm{N}^{(1)}$ (making an angle equal to $\gamma^{(2)}$; see Fig. 1). According to the counterrotation principle, the $d_{y^{\prime} x}$ orbital where the unpaired electron resides is rotated by an angle $-\gamma^{(2)}$ with respect to the same direction. Combining the information given by the angle $\delta$, we have a complete picture of the structure of the active site. Since the direction perpendicular to the imidazole plane is close to $g_{X}$, the imidazole plane is closely perpendicular to the direction $\mathrm{N}^{(1)}-\mathrm{Fe}-\mathrm{N}^{(1)}$ and to the semioccupied orbital. This spatial orientation favors the hypothesis that the repulsion between the $\pi$ cloud of the imidazole and the $d_{x^{\prime} z}$ and $d_{y^{\prime} z}$ orbitals of the iron is the factor determining the orientation of $g_{X}$ in such simple complexes.

\section{Conclusions}

Our study demonstrates that the use of the nuclear quadrupole tensor as a reference frame anchored to the molecule to determine the orientation of nitrogenated ligands and the unpaired electron distribution yields very precise, reliable, and straightforward results. Since the principal values of the heme and axial imidazole $\boldsymbol{Q}$ tensors are not expected to substantially change among LS heme systems, only experiments at the $g=g_{X}$ observer position are strictly necessary to obtain the relative orientations of the heme molecular frame, $\boldsymbol{g}$ tensor, semioccupied orbital, and imidazole plane. Here we have accurately determined the principal values of $\boldsymbol{Q}$ and demonstrated the outstanding performance of the combination of HYSCORE and hyperfine decoupling experiments at $35 \mathrm{GHz}$. Therefore, we anticipate that this method will be widely applied to heme proteins and other heme systems which usually suffer from much lower concentration and where isotope labeling is technically not possible.

Acknowledgments This paper is dedicated to the memory of Arthur Schweiger, who could not see this work completed. The authors want to thank ETH Zürich and the Swiss National Science Foundation for financial support.

\section{References}

1. Milgrom LR (1997) The colours of life: an introduction to the chemistry of porphyrins and related compounds. Oxford University Press, Oxford

2. Moore GR, Pettigrew GW (1990) Cytochrome $c$. Evolutionary, structural and physicochemical aspects. Springer, Berlin

3. Walker FA (1999) Coord Chem Rev 185-186:471-535

4. Bleaney B, Obrien MCM (1956) Proc Phys Soc Lond Sect B 69:1216-1230

5. Griffith JS (1957) Nature 180:30-31

6. Taylor CPS (1977) Biochim Biophys Acta 491:137-149

7. Garcia-Rubio I, Medina M, Cammack R, Alonso PJ, Martinez JI (2006) Biophys J 91:2250-2263

8. Astashkin AV, Raitsimring AM, Walker FA (2001) J Am Chem Soc 123:1905-1913

9. Fahnenschmidt M, Bittl R, Rau HK, Haehnel W, Lubitz W (2000) Chem Phys Lett 323:329-339

10. Fahnenschmidt M, Rau HK, Bittl R, Haehnel W, Lubitz W (1999) Chem Eur J 5:2327-2334

11. Lee HI, Dexter AF, Fann YC, Lakner FJ, Hager LP, Hoffman BM (1997) J Am Chem Soc 119:4059-4069

12. Scholes CP, Falkovski KM, Chen S, Bank J (1986) J Am Chem Soc 108:1660-1671

13. Vinck E, Van Doorslaer S (2004) Phys Chem Chem Phys 6:5324-5330

14. Schunemann V, Raitsimring AM, Benda R, Trautwein AX, Shokireva TK, Walker FA (1999) J Biol Inorg Chem 4:708-716

15. Astashkin AV, Raitsimring AM, Walker FA (1999) Chem Phys Lett 306:9-17

16. Raitsimring AM, Walker FA (1998) J Am Chem Soc 120:9911002 
17. Raitsimring AM, Borbat P, Shokhireva TK, Walker FA (1996) J Phys Chem 100:5235-5244

18. Garcia-Rubio I, Martinez JI, Picorel R, Yruela IL, Alonso PJ (2003) J Am Chem Soc 125:15846-15854

19. García-Rubio I, Alonso PJ, Medina M, Martínez JI (2009) Biophys J 96:141-152

20. Alonso PJ, Martínez JI, García-Rubio I (2007) Coord Chem Rev 251:12-24

21. Byrn MP, Katz BA, Keder NL, Levan KR, Magurany CJ, Miller KM, Pritt JW, Strouse CE (1983) J Am Chem Soc 105:49164922

22. Shokhirev NV, Walker FA (1998) J Am Chem Soc 120:981-990

23. Mitrikas G, Schweiger A (2004) J Magn Reson 168:88-96

24. Van Doorslaer S, Schweiger A (1999) Chem Phys Lett 308:187194

25. Gromov I, Shane J, Forrer J, Rakhmatoullin R, Rozentzwaig Y, Schweiger A (2001) J Magn Reson 149:196-203

26. Gromov I, Forrer J, Schweiger A (2006) Rev Sci Instrum 77:064704
27. Höfer P (1994) J Magn Reson Ser A 111:77-86

28. Schweiger A, Jeschke G (2001) Principles of pulse electron paramagnetic resonance. Oxford University Press, Oxford

29. Ponti A, Schweiger A (1995) J Chem Phys 102:5207-5219

30. Madi ZL, Van Doorslaer S, Schweiger A (2002) J Magn Reson 154:181-191

31. Spaeth JM, Niklas JR, Bartram RH (1992) Structural analysis of point defects in solids. An introduction to multiple resonance spectroscopy. Springer, Berlin

32. Brown TG, Hoffman BM (1980) Mol Phys 39:1073-1109

33. Ashby CIH, Cheng CP, Brown TL (1978) J Am Chem Soc 100:6057-6063

34. Johansson MP, Sundholm D, Gerfen G, Wilkström M (2002) J Am Chem Soc 124:11771-11780

35. Little RG, Dymock KR, Ibers JA (1975) J Am Chem Soc 97:4532-4539 